\title{
Service Level Agreements in Cloud Computing and Big Data
}

\author{
K. Radha ${ }^{1}$, B. Thirumala Rao ${ }^{2}$, Shaik Masthan Babu ${ }^{3}$, K. Thirupathi Rao ${ }^{4}$, V. Krishna Reddy \\ P. Saikiran ${ }^{6}$ \\ 1, 2,4,5,6 Dept. of CSE, KL University, Guntur, India \\ ${ }^{3}$ Sri Sai Educational Societies, India \\ email: radha.saitej@gmail.com¹, thirumail@yahoo.com², bobbycreative.masthan@gmail.com³
}

\begin{tabular}{l} 
Article Info \\
\hline Article history: \\
Received Sep 15, 2014 \\
Revised Nov 30, 2014 \\
Accepted Dec 18, 2014 \\
\hline
\end{tabular}

Keyword:

Analytics

Big Data

Cloud

Distributed Environment

Service Level Agreements

WSLA

\begin{abstract}
Now-a-days Most of the industries are having large volumes of data. Data has range of Tera bytes to Peta byte. Organizations are looking to handle the growth of data. Enterprises are using cloud deployments to address the big data and analytics with respect to the interaction between cloud and big data. This paper presents big data issues and research directions towards the ongoing work of processing of big data in the distributed environments.
\end{abstract}

Corresponding Author:

K Radha,

Departement of Computer Science and Engineering,

KL University,

Green Fields, Vaddeswaram, Guntur, 522502, India

e-mail: radha.saitej@gmail.com

\section{SERVICE LEVEL AGREEMENTS OF CLOUD COMPUTING}

Worldwide enterprises are moving towards Big data to predict the insights from the revolution of information created from different sources [1]. Data is increasing rapidly with the increase in digital world's Petabytes of data from Social Media. Big Data has grown in 2010 US\$3.2 and is expected to grow in 2015 is US $\$ 16.9$ billion [2]. Scale-out uses collection of storage servers to deliver more capacity with same operational cost. In 2020 the universe will produce 50 times of information, 75 times of data storage servers [3]. According to the Gartner report, by 2020, 230 billion devices are connected to the internet. Smart meters, sensors, actuators continuously send massive amount of data that should be analyzed and stored. Today Data Scientists are using Yottabyte to describe how much government data the NASA have on people together. In the near future, Brontobyte will be the measurement to describe the type of sensor data will be generated from the Internet of Things (IoT). $10^{24}$ Yottabyte will be our Digital universe today, (250 trillion of DVDs) and $10^{27}$ Brontobyte will be our digital universe tomorrow. Cloud computing is a business framework on which resources can share the resources over the internet in a pay-per pattern. Due to rapid Growth of data with emerging applications through the Social media, semantic web analysis, Bioinformatics network analysis diversity of data is produced. Managing the data and analysis of massive data is the biggest challenge. Gartner defned Big Data as Huge-Volume, Huge-Velocity and Huge-Variety of data sets are needed in new forms for processing efficient decision-making and process optimization. From 2012-2020, Western Europe’s Digital Universe Growth rate is 538 Exabyes to 5.0 Zettabyte [7].

In the computing world Cloud computing is new business paradigm. According to NIST, Cloud computing is paradigm for allowing pervasive on-demand network access to collection of configurable computational resources that can be providing with service provider interaction. Cloud services provide the 
reliability and manageability in a dynamic world. Cloud computing characteristics are as follows, Rapid elasticity, Pool of Resources, Broad network access, on-demand self service and deliberate service of [1]-[4]. Because of rapid growth of Cloud Computing Market, it is providing the new services with the interaction of cloud service providers and services. Service level agreement is provided for the customers who are utilizing the cloud service to provide the quality of service. In the Dynamic digital environment, services are provided strictly to an on-demand. Agreement is provided among the cloud service providers, brokers, customers. SLA is a legal bond between the service provider and client. Service level Agreement (SLA) has goals through Quality of Service (QoS) attributes, Quality of protection attributes, description of actions to provide the service according to the QoS attributes etc. SLA is designed to create the awareness on QoS and Responsibilities. SLA enables the end-users to agree on what kind of services are offered, how these services will be delivered and who will be responsible for the service execution, service interruptions and privacy aspects. SLA is not providing the assurance about the service expiry [4]. Client organization need to confirm that the authorized data to outside resources should kept to the same high level standards if the data is controlled internally. If there is violation of security the vendor may legally responsible but the client organization may affect. Client organization should understand the business practices with organizational standards.

To host data into the cloud, standard agreements such as confidentiality, service level agreements need to be extended [26]. Cloud computing governance elements are Confidentiality agreements, Nondisclosure, legal location, restricting the software license. Nondisclosure contract is equal to the confidentiality agreement. There is a difference between the confidentiality agreement and Nondisclosure agreement. Nondisclosure agreement need the certifier not truly reveal the information. Confidentiality agreement signatory responsibility is to secure the information. If a dealer has signed on a nondisclosure agreement, if it has security violation, they will not consider as a defect for any revelation whereas in confidentiality agreement, if security breach occurs when a dealer has signed on confidentiality agreement, the dealer should responsible for results of the security violation. Locating the server on network with some specified speed of bandwidth is not an issue from user side but location matters legally. Every user specifies with a service level agreement by the service provider. Dynamic negotiation is occurred when the user needs to specify the exact condition for the requests of users. Weighted sum model (WSM) decides that which provider is offering better user. SLA parameters are scalability, privacy, security, availability. SLA parameters maximize the reliability, confidence level of cloud service provider and cloud user relation. Multi-criteria Decision Making (MCDM) paradigm is used to get the best service provider for cloud use request. This paradigm is used to decrease the service cost to user. This model contains multi-level SLAs for multiple users and dynamic negotiation. Construct a table for every cloud service provider which contains service, data and infrastructure to confirm that which provider offers best service for user's request. Service provider and Infrastructure provider and has connected internally but they have their service level agreements. Service provider would like to run his service in infrastructure to store and to compute. If the infrastructure provider services are accepted by service provider he needs to pay the money to process his service. In this model there is a broker between cloud user and service provider, users will get cost for requested service from all service providers, then compare the cost of all service providers to select less better cost to meet the cloud user request using weighted sum model. Weighted sum model defines that the service provider who meet the Quality of service elements such service, data and deadline for the user. Dynamic model gives optimum cost for the service requested by the user.

Every customer is allocated with service level agreements then investment had set to give the total cost meet the cloud user investment by getting from service provider. When the data is migrating the data from one cloud service provider to the other cloud provider security is an issue. To provide the solution for this issue, Cloud validation is used as a secure storage system for the cloud. Cloud validation based flexible disturbed approach finds the security violations such as integrity, confidentiality. Cryptographic implements involve in looping the signature and simulcast encryption. Simulcast encryption allows migration to encrypt message to random number numb of users. Signature looping is a process of producing consecutive signatures from initial signature and a secret head signature. Interchange of cloud validations secure the data migration process. These validations are essential that allows the users to check the cloud misconduct and cloud providers to protect against violations. Cloud service providers are facing the problem due to uncertainty of workload to reach the service level agreements. Cloud federation paradigm provides service for the cloud users. Cloud users have a choice to select among cloud providers. Common sources of service level agreements violations unexpected interruptions are affected by hardware, software and network failures. Cloud service disruptions are still occurring. Reason for disruptions there are more unknown massive-scale failure situations under which recovery will fail. Failure as a service (FaaS) is proposed to permit the cloud services. This Failure as a Service, cloud services commonly perform massive scale failure in real deployments. Failure as a service is due to high un-stability in distributed environment. As the cloud 
computing resources are growing in research and industry, the possibility of failures affect the applications which are running on the cloud. Failure as a service paradigm is proposed to use by the cloud service providers. Hadoop platform provides application vendors and Hadoop service to test the software towards increase of number failures. Failure as a Service is implemented in Hadoop user's service to evaluate their applications in the presence of failures [28]. Once the computing was done locally it is being done in the cloud. Due to more number of service are thrown unto the cloud, cloud infrastructure size will increase then there is a possibility of failures to be occurred in individual clouds. Applications should not prepare for only the infrequent failures of their cloud infrastructure and they should await the failures as a portion of applications common operating procedure.

At the time of operation, numerous applications and services are running on the cloud will get diverse failures. These failures are ranging from hard disk errors to entire racks. These failures influence greatly on the application performance and sometimes it leads to temporarily out of service. Small sandbox testing is not enough to test the failure effects on real applications which are running on massive number of nodes. Failure as a Service for Hadoop clusters is designed and implemented with Google's Map Reduce for the framework of cloud computing. Failure as a service is used by the cloud service provider and users who will run their services on cloud. Most of the huge enterprises like Amazon, uninterrupted failure paradigm that continually fails diverse parts of their infrastructure to recognize the defect in applications which are running on the cloud. Many developers and organizations cannot access these clouds. To test the failures, small sandbox paradigm is used. Failure as a Service into public Hadoop cloud will permit normal Hadoop users to uninterruptedly inject diverse combination of failures into their cloud applications and also evaluates how they are affected by failures. This will help in identifying the defects in designing their services. Some tools are effectively injecting the failures into cloud software systems such as HDFS. Hadoop contains failure recovery and fault tolerance. Programmable failure injection tool gives failure notions to testers write procedures to eliminate many failure combinations. Failure as a Service is used as quality control tool for organizations and also for users. Evaluate Hadoop in different failure situations by injecting failures at the time of job run times of Network intensive, I/O Intensive and CPU intensive. In virtual service network, Cloud federation provides that users are able to execute the services dynamically provided by the service providers. Allocation of requests from service providers should not be reduced without relevant service level agreements guarantees and without an efficient resource management [29]. Service Level Agreements tools and languages exist either severely limited or too difficult to use by wide set of service providers. Service level agreements can be improved in a less time with suitable tools. Resource management based on the service level agreements is required to agree a pool of virtualized computers inter-connected computers among cloud users and cloud providers. Cloud agency reacts to this benefit added value to actual computing services. As per the available offers it produces SLA that presents the resource negotiation result.

Due to incapability of cloud service providers, they are unable to satisfy the Quality of Service levels are specified in service level agreements which lead to cloud federation vision. This cloud federation is easily act in response to changes in workload, resource and network conditions are aggressively coordinates the numerous clouds in federation. It is impracticable to cloud provider to maintain datacenters in each country, cloud federation provides the added benefit to satisfy the requirements of geologically distributed users than single cloud service providers [27]. Single cloud provision model contains Cloud users are which contains flexible cloud federation model. Cloud federation contains numerous cloud service providers are able to interact among themselves consistently. Cloud computing develops that next generation cloud have ability to form a federation where it will leverages computational resources which reduce the violation of risks of cloud user's service level agreements by moving the jobs between the providers federation. Cloud federation to satisfy the service level agreement acceptance, a middleware layer is needed to coordinate the cloud provider's activities and cloud users activities. Middleware layer should adapt to change the conditions in distributed environment in response to the events that may trigger the service level agreement violation. Middleware needs aggressive scaling to assist heavy workload. Architecture of Cloud federation is proposed which includes a new middleware layer is designed based upon the dynamic data driven application systems model principles. Middle tier chose the cloud service providers to monitor user tasks to assure they are providing within the bounds of service level agreements conditions.

\section{LIFE CYCLE OF SERVICE LEVEL AGREEMENT (SLA)}

SLA life cycle management is divided into five stages such as Service Development, Negotiation and Marketing, Development, Implementation and evaluation. SLA management offers two types of services which are pre-run-time and runtime [5]. Pre-run time refers that, before the service runtime is started, SLA Registration, Service Inquiry and Contract and negotiation has to be done. Service Providers should register the SLA to provide the services to the SLA management system. Service clients will search the services in 
system service library based on QoS needs. Service providers and Service Clients get personalized with each other to negotiate the SLA contract to assure that client can pay as per needs such as SLA metrics and penalty rules. Client should obey the rules given by the service Providers. Run time stage is also called as service operation stage. Run time stage task is to monitor and control the SLA metrics and making the Violation of rules. Make sure that SLA metrics meets the requirements and define the punishment decisions when the rule is violated. SLA offers the Service Providers to differentiate them from the today's competitive environment. SLA and QoS are proposed Web Service Offerings language (WSOL). Terms and Conditions among the organizations and cloud providers set the responsibility at enterprise level. Cloud Computing contracts may set SLA, License agreement to activate on non-performance contract. SLA is good practice to assure the legal areas to seek the appropriate legal guidance. Some of the areas are -obligation with data protection legislation, free of information legislation obligations, confidential information, monitoring the users and security of data, etc. Service Level Agreement Issues are Data protection, Data Security, Location of data, Licensing, Confidentiality, Law and jurisdiction, Retention of data, Direct damages and Indirect damages, Termination [6], [7]. Web Service Level Agreement is proposed to monitor the SLA and to manage the SLAs in a distributed Environment. SLA web services are described as Web Service Level Agreement and Web Service Agreement.

\section{FRAMEWORK OF WEB SERVICE LEVEL AGREEMENT (WSLA)}

Parties, SLA parameters, Service Level Objectives (SLOs) are three entities of Web Service Level Agreement [8]. There are three types of Parties namely Service Provider, Service Consumer and Third Parties. Third parties may vary to take decisions on violations either by the Service Provider or Service Consumer. In Web Service Level Agreements (WSLA), SLA parameters are used to measure the service parameters. There are two kinds of Metrics such as Composite metrics and Resource metrics. From the service provider's resources resource Metrics are accessed. Composite Metrics are combination of various resource metrics and Service Level Objectives are set of expressions as if-then structure. Web services are provided to the consumers with different service levels by suing the automated management and service level agreements. Web service level agreement language specifies that to supply the resources based on the SLA. Workload management gives the priority to the requests associated with service level agreements and to monitor the agreement with the service level agreement. Principles of The WSLA Framework are SLA Parameters, Business Metrics, Resource Metrics, and Composite Metrics. Resource metrics are directly accessed from the controlled resources which are there in the service provider's tier such as routers and servers. Composite metrics are generated by gathering various resources. Composite metrics are defined by service level agreements. Business metrics form the consumer risk management policy which is available within the customer service domain. Service provider performs a mapping to assure that service level agreement and to fulfill business goals. Web service level agreement language is XML schema.

\section{PRESENT ANATOMY OF CLOUD SERVICE AGREEMENT}

Cloud Consumers compare the agreements between the distinct public cloud providers. A consumer has to be careful while selecting the language for the agreements. Sometimes there are vocabulary errors that alter the meaning of a clause. Before signing the contract catch the errors and correct the errors. Cloud agreement is divided into three types such as End-User agreement, Accepting the Policies and Service Level Agreement [9]. In End-User Agreement, Business service management consists the policies of cloud providers. Customer Agreement fulfills the requirement of “Terms of Service”. Public cloud customer agreements consists of the critical sections as follows offered services, breaking the service temporarily, paying the fee, terminating the terms and conditions, disclaimer, limited liability and security. Offered services describes that, how customer utilizes the public cloud offerings in terms of supplying the service and Service Description details. Payment of fee refers that, it is the method of spending the fee for the cloud services such as Service Charge Schedule, buying the service and defrayment Terms and Conditions. Temporary suspension of a service is a process where the service provider suspends the usage of the cloud by specific consumer for a time based on the drawback of anomalous usage of distributed environment and safety problems.

Terminating the Terms and Conditions describes that Service providers terminate services used by the client and Closes the Account of clients. Disclaimer is description of services which are not included in the agreement in terms of Warranties and Disclaimer. Limited liability specifies a limit that a customer can claim on the limited payment. Security includes that Responsibility, protecting the Data, Privacy plan and Customer responsibility. Acceptable Use Policies refers that Public cloud providers and Cloud Clients should agree the terms and Conditions. In Service Level Agreements of Cloud phase, Service Level Agreements of 
Cloud are accepted for both Client and provider that defines the set of objectives contains availability, performance, Security and privacy policies.SLA Challenges are SLA Architecture, SLA Based Scheduling Polices, and Resource Allocation of SLA. Throughout the worldwide, Cloud computing offers paymentoriented and organizational-Quality computing services to end-users. Service Providers deploy data processing centers in various locations for providing backup for ensuring the reliability.

\section{CHALLENGES OF SERVICE LEVEL AGREEMENTS (SLA)}

Resource management issues are involved in delivering the services for a millions of user's services via data center. Challenges of service level agreements are as follows data processing of risk management, consumer-driven service management, and independent resource management, measuring the service, system design and reiteration valuation resource allocation in SLA with virtualization.

\subsection{Consumer-Driven Service Management}

Three user-centric objectives are used to satisfy the customer requirement which includes getting the feedback from the customers, providing the reliable communication between the customers, increasing the access efficiency to understand the specific necessities of the customer and believe the customer. When the service is developing, if customer expectations are considered, then those expectations of customer are imported into service provider. If these expectations are implemented by service provider should satisfy the client's requirements. Reasonable expectations of customer are accepted and adopted by the provider then it enters into customer contract. Customer-driven contracts characteristics are as follows, singular, onauthoritative, bounded stability and immutability complete and closed. Customer-driven agreement is completed and closed to the complete collection of functionality demanded by existing customers. Provider agreements are single in their expression of business functionality available to the system, whereas nonauthoritative are derived from combination of existing consumer expectations. Customer driven agreement is unchangeable with respect to the particular set of customer agreements. Validity of customer driven agreement according to a specified set of customer agreements is effectively bounded to the forward and backward compatible agreement in space and time. The compatibility of consumer agreement remains unchanged for specified customer expectations.

\subsection{Data Processing of Risk Management}

The Risk Management process contains the following ways such as Identifying the Risk and assesses the risk, identify the techniques to manage the risks and review the risk management plan. Quality of service conditions of grid service customers need the form of service level agreements among users and service providers. Hazards for a Successful service level agreement supplying in grid computing, firstly failures of computational nodes. Grid service providers require the risk analysis to evaluate expected losses in resource management. Deciding that whether to accept or reject service level agreement request is an issue for service providers, since resource are disrupted and unavailable hazard [31].

\subsection{Independent Resource Management}

Data processing center should maintain the reservation process without interruption by managing the present service requisition and improve the future service requisition and altering the price for the newly received requests [10]. SLA Risk Management Challenges are Information Security and Risk Management, SLA and Exception management [11], [12]. Developing SLA in Data Center Service Level agreements helps that the IDC Promises that what is Possible to deliver, delivers what IDC is promised [15]. Basic problem with cloud applications is to compare the tasks namely as follows data transmission or computation into a pool of resources that should meet the cloud applications cost, performance, security parameters. In resource management paradigm, interactions of resources are mapped to a pool of platform independent service level agreements [30]. Service level agreements do not guarantee the response time and also it is difficult because of unpredictable traffic patterns. Adaptive resource management increases the usage of web application by maximizing the resource utilization. Architecture of resource management with the cooperation of computing systems via numerous virtual machines increases the performance of computational systems will improve the utilization of resources designed for on-demand utilization of resource. Architecture of resource management has benefits of some components in virtualized platform, cloud computing platform and grid computing platform reduces the overhead of computational systems. Architecture of resource management with the cooperation of computing systems has highest CPU utilization and best performance. Virtual machine based resource provisioning is adopted in distributed environments. Allocations of virtual machines by using static scheduling mechanism, resources are not completely utilized. Virtual machine uses optimal cloud resource provisioning in dynamic resource allocation. Service level agreements should be used to give trading of 
resources based upon the economy models. Service level agreements are able to find their needs and identify the provider abilities. Customer driven agreements has two advantages such as delivery and specification of service functionality around the business value drivers and minimal set of requirements are develop the service when customer expect a service to develop, operate and deploy in a manageable manner. Customer driven agreement is suitable to single organization. These agreements efficiently control the interrupted changes to the agreements. This agreement optimizes the bounding between the services.

\subsection{Resource Allocation in SLA with Virtualization}

Virtualization provides computing resources. Hence a isolated physical machine is capable to work like many logical Virtual Machines. Virtual machines are able to provides multiple operating system environments and capable to configure Virtual Machines to use distinct segregation of resources on the same physical machine. Virtual machines assigned various resource management policies are providing for the various user requirements to support the implement resource allocation in SLA. Supplying the resources efficiently is a challenging problem in distributed environment because of its Changing nature and necessity of supporting the diverse applications with distinct performance requirements. Resource allocation problem in data center provides various kinds of application workloads specifically reciprocated and enterprise applications. Admission control and scheduling mechanism is proposed for utilizing the resources and enduser SLA needs [25]. An optimal joint multiple resource allocation method is used in Allocation of resource model of distributed environment. The resources which Allocated are committed to every service request. These methods are decrease the probability of requisition loss and reduce the total resource. Resource allocation approach is proposed for the multi-dimensional resource allocation problem to execute user's applications.

\subsection{Measuring the Service}

Various Service providers are providing distinct computing services. Service measurement acquires most suitable services to satisfy the consumer needs. Evaluating the service performance is required for original cloud footprints from various public documents to model the application and service needs. Presently there are no measurements are available to determine utilization-based monitoring of resources for the distributed environment. There are various data-intensive applications and workflow applications, reliability and security. There is necessity to predict the collection of service measurements for exact assessment of controlling the resource principles. Benchmark provides the future prediction of consumer requirements. Service measurement has information on the configuration of the current system and runtime information metrics as part of the service level agreement. It meters the parameters of service level agreement by directly accessing form managed resources [32]. Measureable qualities of service level agreements are quality, availability, cost, capacity and latency whereas un-measureable qualities are security, interoperability and modifiability.

\subsection{System Design and Reiteration Valuation}

Resource management plans are evaluated through different kinds of sources and consumers with variant service prerequisite to prove the efficiency. It is tedious to perform performance assessment of monitoring the resource plans in repetition and administrable fashion since resources are transferred and service requisitions will come from various consumers at any stage. Monitoring the resource strategies performance can be evaluated by Discrepancy-event simulation. CloudSim estimate the resource monitoring plans performance. CloudSim is a tool kit is used to model and simulation of distributed environment resources and scheduling the applications. ClouSim construct the simulation frameworks to calculate the performance of management of resource policies [24]. Utility Computing provides subscription-oriented services. SLA components are Scope, Limitations, Validity, Purpose, Parties, Service Level objectives (SLO) Penalties, Optional Services, Administration. SLAs are created, monitored and utilized in utility computing environment [16].

\section{SERVICE LEVEL AGREEMENT FOR SERVICE-ORIENTED SYSTEMS}

Service-oriented architecture is to design and built the Service Bases Systems. SLA should specify the Quality of Service (QoS) related to specific roles. Service Based systems provide value-added services. Web Service Description Language (WSDL), Simple Object Access Protocol (SOAP) technologies are used by Service-based systems. There are three issues such as SLA life cycle management, SLA violation-impact analysis, SLA verification and Generation, [17]. Cloud computing systems are Utility computing reliable data storage systems. Each Consumer has Service Level Agreement specifies that, it has obligation on performance and service's Quality that is retrieved from the system. Cloud Computing system pays 
penalization if the consumer's requests are not satisfied on their service time [18]. Various rules are introduced for Business Rules for maximizing the Revenue of Service provider's in cloud computing for Negotiation and execution time to achieve the business level objectives among the market and resource layers. Cloud Performance is added To Measure and Use the QoS to determine the availability, liability in case of failure [20], [21]. Cloud service Brokering allows to access the Cloud Resource continuously among the Consumers and Service providers, management and monitoring of deployed cloud services on various Cloud service providers finally Cloud resources should satisfy the Customers those are specified by SLA [22]. Main purpose of the service-oriented model is managing and controlling the evolution of services. Service-oriented model uses the services to develop the less cost, evolvable, interoperable and magnitude of applications. Services can be discovered, published, described. Service-oriented mechanism discovers and invokes the network available services to complete some task. Service oriented engineering and service model is essential to create semantic services and business process specifications. Service-oriented mechanism discovers and invokes the network available services to complete some task.

Best practices are used to describe the process developing that can be used multiple partners. Best practices are as follows Identifying the Cloud Actors, Evaluation of business level policies, Metrics, Security, Identifying service management requirements, Prepare and manage service failures [14], [19].

\section{SERVICE LEVEL AGREEMENTS IN BIG DATA}

The data growing rapidly by the consumers, business and government generated content. To maintain this data there are rules with service level agreements to protect the data. Capacity, scalability, security, privacy, availability are the issues of data storage and data growth [23]. In service oriented architecture environment quality attribute needs play major role in selecting the service. Specification of service level agreements provides that to ensure the services are provided with availability, security, performance.

\section{CONCLUSION}

Due to rapid growth of Cloud Computing Market, it is providing the new services with the interaction of cloud service providers and services. Service level agreement is provided for the customers who are utilizing the cloud service to provide the quality of service. SLA web services are described as Web Service Level Agreement and Web Service Agreement. Web Service Level Agreement is divided into three entities such as Parties, SLA parameters, Service Level Objectives (SLOs). Challenges of Service level agreements (SLA) as Consumer-driven Service Management, Data processing of risk management, Independent Resource management. Service level agreements are implemented in Service oriented architecture.

\section{REFERENCES}

[1] Peter Mell, Timothy Grance, “NIST Definition of Cloud Computing”, Special Publication 800-145.

[2] Judith Hurwitz, Robin Bloor, Marcia Kaufman, Cloud Computing for dummies, HP Special edition.

[3] C.N.Hoefer, G. Karagiannis, "Taxonomy of cloud computing services”.

[4] Brussels, “Cloud Computing Service Level Agreements”, June 2013.

[5] Shu Zhang, Meina Song, Junde Song, “A Life Cycle Based SLA Management Architecture Design”.

[6] "User Guide: Cloud Computing Contracts, SLAs and Terms \& Conditions of Use”, August 2011.

[7] Steve McDonal, "Legal and Quasi-Legal Issues in Cloud Computing Contracts”.

[8] Pankesh Patel, Ajith Ranabahu, Amit Sheth, "Service Level Agreement in Cloud Computing".

[9] "Public Cloud Service Agreements": What to Expect and What to Negotiate, March 30, 2013.

[10] Rajkumar Buyya, Saurabh Kumar Garg, Rodrigo N. Calheiros, "SLA-Oriented Resource Provisioning for Cloud Computing: Challenges, Architecture, and Solutions".

[11] Jean-Henry Morin, Jocelyn Aubert, Benjamin Gateau, “Towards Cloud Computing SLA Risk Management: Issues and Challenges"

[12] Mitchell Cochran, Paul d. Witman, "Governance and service level agreement issues in a Cloud computing environment".

[13] "Creating Effective Cloud Computing Contracts for the Federal Government", Federal Cloud Compliance Committee, February 24, 2012.

[14] Judith M. Myerson, “Best practices to develop SLAs for cloud computing”, 07 January 2013.

[15] Edward Wusten Hoff, "Service Level Agreement in the Data Center".

[16] Linlin Wu, Rajkumar Buyya, Service Level Agreement (SLA) in Utility Computing Systems.

[17] Azlan Ismail, “Temporal-aware Service Level Agreement management for Service-based systems”. 
[18] Hadi Goudarzi, Mohammad Ghasemazar, assoud Pedram, "SLA-based Optimization of Power and Migration Cost in Cloud Computing".

[19] Andrew Geyer, Melinda McLellan, Hunton, Williams LLP, "Strategies for Evaluating Cloud Computing Agreements"

[20] Mario Mac' ıas, J. Oriol Fit'o and Jordi Guitart, "Rule-based SLA Management for Revenue Maximization in Cloud Computing Markets".

[21] Lee Gillam, Bin Li, and John O'Loughlin, “Adding Cloud Performance to Service Level Agreements”.

[22] Fouled Jrad, Jie Tao and Achim Streit, "SLA based service brokering in intercloud environments".

[23] Alnafoosi, A.B, Steinbach, T. An integrated framework for evaluating big-data storage solutions - IDA case study, Science and Information Conference (SAI), 2013,947 - 956

[24] S.K. Garg and R. Buyya. Network Cloud Sim: Modeling Parallel Applications in Cloud Simulations. Proceedings of the 4th IEEE/ACM International Conference on Utility and Cloud Computing (UCC 2011), Melbourne, Australia, December, 2011

[25] S.K. Garg, S.K. Gopalaiyengar, and R. Buyya. "SLA-based Resource Provisioning for Heterogeneous Workloads in a Virtualized Cloud Datacenter". Proceedings of the 11th International Conference on Algorithms and Architectures for Parallel Processing (ICA3PP 2011), Melbourne, Australia, October, 2011.

[26] Mitchell Cochran, Paul d. witman, "governance and service level agreement issues in a cloud computing environment", a publication of the association of management.

[27] Funmilade Faniyi, Rami Bahsoona, Georgios Theodoropoulos, “A Dynamic Data-Driven Simulation Approach for Preventing Service Level Agreement Violations in Cloud Federation”, International Conference on Computational Science, ICCS 2012.

[28] Sobir Bazarbayev, Faraz Faghri, Mark Overholt, "Failure as a Service (FaaS) for Hadoop Cluster".

[29] P. Rubach, M. Sobolewski, Autonomic SLA management in federated computing environments, in: Proceedings of the 2009 International Conference on Parallel Processing Workshops, ICPPW '09, IEEE Computer Society, Washington, DC, USA, 2009, pp. 314-321.

[30] Karl Czajkowski, Ian Foster, Carl Kesselman, Volker Sander, Steven Tuecke, "SNAP: A Protocol for Negotiating Service Level Agreements and Coordinating Resource Management in Distributed Systems”, In 8th Workshop on Job Scheduling Strategies for Parallel Processing.

[31] D. Battr_e, G. Birkenheuer, et al,” Applying Risk Management to Support SLA Provisioning”.

[32] Alexander Keller, Heiko Ludwig, "The WSLA Framework: Specifying and Monitoring Service Level Agreements for Web Services".

\section{BIOGRAPHIES OF AUTHORS}
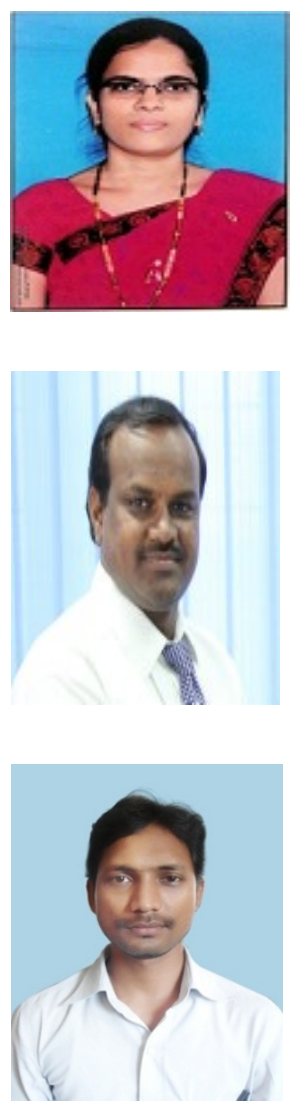

K. Radha Pursuing PhD from KL University, Guntur. Completed M.Tech from Anurag Engineering College, Kodad and B.Tech Degree from G. Narayanamma Institute of Technology \&Sciences for Women, Hyd. Worked as an Assistant Professor for 6 years in Laqshya Instiute of Technology and Sciences, Khammam.

Dr. B. Thirumala Rao is working as a Professor in KL University, Guntur. Ph.D. in Computer Science \& Engineering in the area of Cloud Computing from Acharya Nagarjuna University .M.Tech (CSE) from Jawaharlal Nehru University, Hyderabad. B.Tech (CSE) from Acharya Nagarjuna University.

Shaik Masthan Babu working as an Assistant Professor in Sri Sai Educational Society’s since 6 years and Pursuing PhD in KL University, Guntur. M.Tech from Anurag Engineering College, Kodad. B.Tech from Khader Memorial College of Engineering, Devarakonda. 